\title{
Nearfield target localization with a few snapshots for sonar array
}

Zhuo Li, ${ }^{1,2}$, Deliang Liü ${ }^{3^{*}}$, Xiwei Guo ${ }^{3}$ and Peng $\mathrm{He}^{3}$

\begin{abstract}
Sonars can be used for detection of targets located on the sea floor where the signal undergoes multipath propagations which may arise large localization errors. Besides, underwater environment is often highly unstable so that a large number of snapshots which means long time sampling are not reliable. In sonar systems, the wideband chirp signals are usually used. This paper presents a nearfield target localization method for underwater environments. First, the time of arrival (TOA) and the direction of arrival (DOA) for nearfield wideband chirp signals are estimated with only a few snapshots by using the fractional Fourier transform (FRFT) and the SPICE algorithm. Then, the virtual station method which is proposed in our previous work is used so that the multipath problems can be converted into line-of-sight (LOS) problem and its Cramer-Rao lower bound (CRLB) is also analyzed. Finally, simulation results demonstrate the effectiveness of the proposed method.
\end{abstract}

Keywords: Sonar, Target localization, Multipath, Nearfield

\section{Introduction}

Sonar systems have been widely used for decades to detect an underwater target [1-3]. The active sonar transmits an acoustic signal at a predetermined interval and then receives signals using a sensor array to find echoes that are reflected from a target, while the passive sonar which contains sensor array receives the acoustic signal which is transmitted from the target. The received signal usually contains an echo signal, ambient noise, and reverberation signals induced by underwater environments [4-6] which is also called multipath problem. The conventional localization methods for free space environment are limited in underwater environment because of the multipath signal propagation. To mitigate these impacts, a variety of algorithms have been proposed in the literatures. A detection scheme in [7] is used to detect and discard multiple-bound scattering paths, which will not work when all signal paths are multiple-bound scattering paths. Joint iterative phase reconstruction and weighted localization algorithm is used in [8], but the transmitted and received signal paths at the same reader may be different which may cause large errors.

\footnotetext{
* Correspondence: liudeliang82@sina.com

${ }^{3}$ Army Engineering University, Shijiazhuang 050003, China

Full list of author information is available at the end of the article
}

Robust schemes in $[9,10]$ derive the line of possible mobile device (LPMD) but they cannot apply to the multiple-bound scattering conditions. In our previous work [11], a virtual station (VS) method has been proposed to solve the multipath problem and it will be directly used in this paper.

Another challenge emerges when the number of snapshots available for the estimation of the location parameters such as time of arrival (TOA) and direction of arrival (DOA) is limited. In underwater conditions, a large number of snapshots are not reliable due to the motion of the target and the highly nonstationary of the environment which is caused by complex electromagnetic environment and the people who appear randomly. Therefore, the performance of the conventional array processing methods, such as MUSIC and ESPRIT, which require a large amount of snapshots to provide super-resolution DOA and TOA estimation, will be dramatically degraded. Several methods have been proposed in the literature to solve this problem. IAA, SLIM, and SPICE algorithms [12] are sparsity-based techniques that can estimate the DOA depending on a few snapshots with high resolution and low sidelobes. But they only apply to narrowband signals, because the steering vector of the received wideband signal model is time-variant. So 
some extensions to this method have been proposed to deal with the wideband signals, such as spatial resampling (SR) method [13], the accuracy of which is not high due to using an approximate model for wideband chirp signals.

In this paper, a joint TOA and DOA localization method for wideband chirp signals which is able to locate underwater targets with only a few snapshots is proposed. First, wideband chirp signal was transformed with a specific transform order into sine wave with a single frequency in fractional Fourier transform (FRFT) domain, so that the time-invariant steering vector of the wideband chirp can be obtained. Then, the SPICE algorithm is utilized to estimate the TOA and DOA of the targets with two snapshots. Finally, the VS method is used to estimate the target location using the hybrid TOA and DOA values and its Cramer-Rao lower bound (CRLB) is also analyzed.

The rest of this paper is organized as follows. In Section 2, the SPICE method is developed in the FRFT domain to estimate the nearfield TOA and DOA of wideband chirp signal. In Section 3, the VS method which utilizes the TOA and DOA measurements is directly used and the CRLB is also analyzed. Section 4 presents the experimental results. The paper is concluded in Section 5.

\section{Joint DOA and TOA estimation with a few snapshots}

\subsection{Sonar array model}

Considering a passive sonar array system as shown in Fig. 1. A nearfield model is used in this paper. The linear array of the sonar has $M$ sensors uniformly placed along the $x$-axis. The distance between two adjacent sensors is $d . \mathrm{T}_{1}, \mathrm{~T}_{2}, \ldots, \mathrm{T}_{\mathrm{K}}$ are $\mathrm{K}$ targets at $[\boldsymbol{r}$, $\boldsymbol{\theta}]$, where $\boldsymbol{r}=\left[r_{1}, r_{2}, \ldots, r_{\mathrm{K}}\right]$ represent the distances of the targets and $\boldsymbol{\theta}=\left[\theta_{1}, \theta_{2}, \ldots, \theta_{\mathrm{K}}\right]$ represent the directions of the targets. Here, $K$ is usually unknown, so it

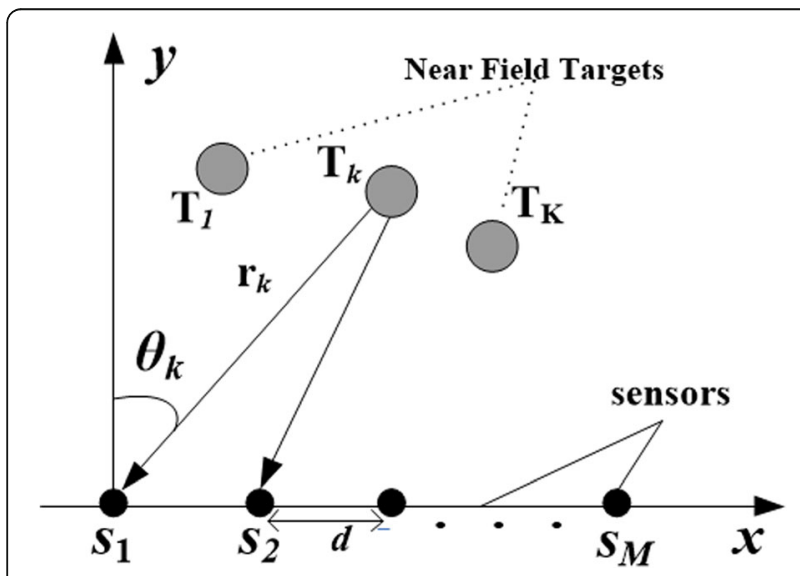

Fig. 1 Passive sonar array model is considered to be the amount of potential targets (scanning points) in the region and it is much larger than the amount of actual ones. Only a few signal power estimates of the potential targets will be nonzero, so sparsity-based algorithm can be used in array processing applications.

The target emits a chirp signal that can be expressed as [14]

$$
x(t)=a \exp \left(j 2 \pi f_{0} t+j \pi \mu t^{2}\right)
$$

where $a$ is the signal amplitude, $f_{0}$ is the center frequency, and $\mu$ is the chirp rate.

The received signal at the sensor $m$ can be expressed as the sum of $K$ delayed versions of $x(t)$, given by [14]

$$
x^{m}(t)=\sum_{k=1}^{K} \rho_{k} x_{k}\left(t-\tau_{k}^{m}\right)+e^{m}(t)
$$

where $e^{m}(t)$ for $m=1,2, \ldots, M$ is the additive white Gaussian noise at the sensor $m, \rho_{\mathrm{k}}$ is the path loss coefficient of target $k$ for $k=1,2, \ldots, K . \tau_{k}^{m}$ is the time delay of the $k$ th signal traveling to the sensor $m$ relative to the reference sensor (the first sensor), which can be expressed as [14]

$$
\tau_{k}^{m}=\frac{r_{k}-\sqrt{\left[r_{k} \sin \theta_{k}-(m-1) d\right]^{2}+r_{k}^{2} \cos \theta_{k}}}{c}
$$

where $c$ is the wave speed.

Ignoring the high-order terms from the Taylor expansion, Eq. (3) can be given as [14]

$$
\tau_{k}^{m} \approx \frac{-(m-1) d \sin \theta_{k}+\frac{1}{2}(m-1)^{2} d^{2} \cos ^{2} \theta_{k} / r_{k}}{c}
$$

The received signals at the sensors can be written in matrix form as [14]

$$
\mathbf{x}(t)=\mathbf{a s}(t)+\mathbf{e}(t)
$$

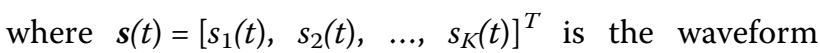
vector and $\boldsymbol{a}=\left[\boldsymbol{a}\left(\theta_{1}\right), \boldsymbol{a}\left(\theta_{2}\right), \ldots, \boldsymbol{a}\left(\theta_{K}\right)\right]^{T}$ is the steering vector with

$$
\begin{aligned}
& \mathbf{a}\left(\theta_{k}\right)=\left[\exp \left(-j 2 \pi f_{0} \tau_{1}+j \pi \mu \tau_{1}^{2}\right) \exp \left(-j 2 \pi \mu \tau_{1} t\right), \ldots,\right. \\
& \left.\exp \left(-j 2 \pi f_{0} \tau_{M}+j \pi \mu \tau_{M}^{2}\right) \exp \left(-j 2 \pi \mu \tau_{M} t\right)\right]^{T}
\end{aligned}
$$

When the narrowband signal is used in the system, $\boldsymbol{a}\left(\theta_{k}\right)$ can be considered as time-invariant (i.e., the term exp. $\left(-j 2 \pi \mu \tau_{\mathrm{m}} \mathrm{t}\right)$ in (6) can be neglected). In this paper, we focus on wideband chirp, so the steering vector $\boldsymbol{a}\left(\theta_{k}\right)$ depends on the time $t$, and the SPICE algorithm for narrowband signals cannot be applied 
directly to wideband signals. To solve this problem, we derive the FRFT method.

\subsection{The FRFT of wideband chirp}

The FRFT of $x(t)$ in (1) about angle $\alpha$ can be represented as

$$
\begin{aligned}
X(\alpha, u) & =a \sqrt{\frac{1+j \tan \alpha}{1+\mu \tan \alpha}} \\
& \times \exp \left[j \pi \frac{u^{2}(\mu-\tan \alpha)+2 u f_{0} \sec \alpha-f_{0}^{2} \tan \alpha}{1+\mu \tan \alpha}\right]
\end{aligned}
$$

When $\alpha=\alpha_{d}=\arctan \left(\mu s_{c}{ }^{2}\right)$, we can get

$$
X\left(\alpha_{d}, u\right)=C \exp \left[j 2 \pi f_{0} \cos \alpha_{d} u\right]
$$

where $s_{c}=\sqrt{N} / f_{s}$ is the scale factor to normalize signals [15] with $N$ which denotes the number of snapshots and $f_{s}$ is the sampling frequency. $C=a \cos \alpha_{d} \sqrt{1+j \tan \alpha_{d}} \exp \left[-j \pi f_{0}^{2} \sin \alpha_{d} \cos \alpha_{d}\right]$ is a constant. By taking the FRFT, the chirp signal becomes a sine wave with a single frequency $f=f_{0}$ $\cos \alpha_{d}$.

At the sensor $m$, the received chirp from target $k$ in the FRFT domain according to the time shift characteristic of FRFT can be given as

$$
\begin{aligned}
X_{k}^{m}\left(\alpha_{d}, u\right) & =F^{p}\left[x\left(t-\tau_{k}^{m}\right)\right] \\
& =\rho_{k} a C \exp \left[j 2 \pi\left(f_{0} \cos \alpha_{d}-\tau_{k}^{m} \sin \alpha_{d}\right) u\right] \\
& \times \exp \left\{j \pi\left[\left(\tau_{k}^{m}\right)^{2} \sin \alpha_{d} \cos \alpha_{d}-2 f \cos ^{2} \alpha_{d} \tau_{k}^{m}\right]\right\}
\end{aligned}
$$

Because $\tau_{k}^{m} \sin \alpha_{d}$ is very small, we can get

$$
\begin{aligned}
X_{k}^{m}\left(\alpha_{d}, u\right) & \approx \rho_{k} a C \exp \left(j 2 \pi f_{0} \cos \alpha_{d} u\right) \\
& \times \exp \left\{j \pi\left[\left(\tau_{k}^{m}\right)^{2} \sin \alpha_{d} \cos \alpha_{d}-2 f \cos ^{2} \alpha_{d} \tau_{k}^{m}\right]\right\}
\end{aligned}
$$

Therefore, the FRFT of (5) can be given as

$$
\mathbf{X}=\mathbf{A S}+\mathbf{E}
$$

where

$$
\begin{aligned}
\mathbf{S} & =\operatorname{diag}\left\{S_{1}(u), S_{2}(u), \ldots, S_{K}(u)\right\}, \mathbf{X} \\
& =\left[\mathbf{X}^{1}, \mathbf{X}^{2}, \ldots, \mathbf{X}^{M}\right]^{T}, \mathbf{A}=\left[\mathbf{A}_{1}, \mathbf{A}_{2}, \ldots, \mathbf{A}_{K}\right]^{T}
\end{aligned}
$$

with

$$
\begin{aligned}
& \mathbf{X}^{m}=\left[X_{1}^{m}\left(\alpha_{d}, u\right), X_{2}^{m}\left(\alpha_{d}, u\right), \ldots, X_{K}^{m}\left(\alpha_{d}, u\right)\right]^{T}, \\
& \mathbf{A}_{k}=\left[A_{k}^{1}\left(\theta_{k}\right), A_{k}^{2}\left(\theta_{k}\right), \ldots, A_{k}^{M}\left(\theta_{k}\right)\right]^{T}
\end{aligned}
$$

$$
\begin{aligned}
& A_{k}^{m}=\exp \left\{j \pi\left[\left(\tau_{k}^{m}\right)^{2} \sin \alpha_{d} \cos \alpha_{d}-2 f \cos ^{2} \alpha_{d} \tau_{k}^{m}\right]\right\} \\
& S_{k}(u)=\rho_{k} a C \exp \left(j 2 \pi f_{0} \cos \alpha_{d} u\right)
\end{aligned}
$$

$\mathbf{E}$ in (11) is the FRFT of $\mathbf{e}$ in (5) which is usually assumed to be white Gaussian noise. The power spectrum density of a random signal $x(t)$ in FRFT domain with angle $\alpha$ is

$$
P(\alpha, u)=\lim _{T \rightarrow \infty} \frac{E|X(\alpha, u)|^{2}}{2 T}
$$

The observation time is $[-T, T]$.

Considering the relationship between the power spectrum density and the autocorrelative function, we can get

$$
\begin{aligned}
& P(\alpha, u)= \lim _{T \rightarrow \infty} \frac{1}{2 T} E\left[A_{\alpha} \int_{-T}^{T} x\left(t_{1}\right) \exp \right. \\
&\left.\times A_{-\alpha} \int_{-T}^{T} x^{*}\left(t_{2}\right) \exp \left(-j \pi t_{2}^{2} \cot \alpha+2 j \pi t_{2} u \csc \alpha-j \pi u^{2} \cot \alpha\right) d t_{2}\right] \\
&= \lim _{T \rightarrow \infty} \frac{1}{2 T} E\left\{A_{\alpha} A_{-\alpha} \int_{-T}^{T} d t_{1} \int_{-T}^{T} \exp \left[j \pi \cot \alpha\left(t_{1}^{2}-t_{2}^{2}\right)\right] \exp \right. \\
& {\left.\left[-j 2 \pi\left(t_{1}-t_{2}\right) u \csc \alpha\right] x\left(t_{1}\right) x^{*}\left(t_{2}\right) d t_{2}\right\} }
\end{aligned}
$$

where $A_{\alpha}=\sqrt{1-j \cot \alpha}$.

Making $t_{1-} t_{2}=\tau$, we can get

$$
\begin{aligned}
P(\alpha, u)= & A_{\alpha} A_{-\alpha} \lim _{T \rightarrow \infty} \frac{1}{2 T} \int_{-T-t_{2}}^{T-t_{2}} d \tau \int_{-T}^{T} \\
& \exp \left[\left(2 j \pi t_{2}+j \pi \tau\right) \tau \cot \alpha\right] \exp (-j 2 \pi \tau u \csc \alpha) R\left(t_{2}+\tau, t_{2}\right) d t_{2} \\
= & A_{\alpha} A_{-\alpha} \int_{-\infty}^{\infty} \exp (-j 2 \pi \tau u \csc \alpha) \exp \left(j \pi \tau^{2} \cot \alpha\right) \\
& \quad\left[\lim _{T \rightarrow \infty} \frac{1}{2 T} \int_{-T}^{T} \exp \left(j 2 \pi t_{2} \tau \cot \alpha\right) R\left(t_{2}+\tau, t_{2}\right) d t_{2}\right] d \tau \\
= & A_{-\alpha} \exp \left(-j \pi u^{2} \cot \alpha\right) F^{\alpha}[R(\alpha, \tau)](u)
\end{aligned}
$$

where

$$
R(\alpha, \tau)=\lim _{T \rightarrow \infty} \frac{1}{2 T} \int_{-T}^{T} \exp \left(j 2 \pi t_{2} \tau \cot \alpha\right) R\left(t_{2}+\tau, t_{2}\right) d t_{2}
$$

When $P(\alpha, u)=N_{0}, x(t)$ is the white noise in FRFT domain with angle $\alpha$.

Considering the correlation function of white noise in the FRFT domain, we can get

$$
R(\alpha, \tau)=\frac{N_{0} \exp \left(-j \pi \tau^{2} \cot \alpha\right)}{\csc \alpha} \delta(\tau)
$$

According to the definition of FRFT, the power spectrum density in the FRFT domain with angle $\beta$ of 
the white noise in FRFT domain with angle $\alpha$ can be given as

$$
P(\beta, u)=N_{0}
$$

We can see that the power spectrum density in the FRFT domain of the white noise is changeless, so the FRFT will not change the statistical characteristics of the white noise, and E in (17) is still Gaussian.

\subsection{Joint TOA and DOA estimation by SPICE}

The discrete form of (11) can be given as

$$
\mathbf{X}(n)=\mathbf{A S}(n)+\mathbf{E}(n)
$$

where $n=1,2, \ldots, N$ is the number of snapshots.

Let $\mathbf{P}$ be a $K \times K$ diagonal matrix, whose diagonal contains the power at each angle on the scanning grid. The initial estimates $\hat{\mathbf{P}}_{k}^{(0)}$ can be obtained using the SFLS method [16]

$$
\hat{\mathbf{P}}_{k}^{(0)}=\frac{\sum_{n=1}^{N}\left|\mathbf{A}_{k}{ }^{H} \mathbf{X}(n)\right|^{2}}{\left(\mathbf{A}_{k}{ }^{H} \mathbf{A}_{k}\right)^{2} N}
$$

The noise covariance matrix $\mathbf{R}$ of $\mathbf{X}(\mathrm{n})$ can be given as

$$
\mathbf{R}^{(i)}=\mathbf{E}\left(\mathbf{X} \mathbf{X}^{H}\right)=\mathbf{A} \hat{\mathbf{P}}^{(i-1)} \mathbf{A}^{H}
$$

Then, in the $i+1$ iteration, the power at each angle on the scanning grid can be updated as

$$
p_{k}^{(i+1)}=\frac{\left|\beta_{k}^{(i)}\right|}{w_{k}^{1 / 2} \sum_{l=1}^{K} w_{l}^{1 / 2}\left|\beta_{l}^{(i)}\right|}
$$

where

$$
\begin{gathered}
w_{k}=\frac{\left\|a_{k}\right\|^{2}}{\operatorname{tr}(\mathbf{R})}, \\
\left|\beta_{k}\right|^{2}=\left[\mathbf{Q X}\left(\mathbf{X}^{H} \mathbf{X}\right) \mathbf{X}^{H} \mathbf{Q}^{H}\right]_{k k} / N^{2}, \\
\mathbf{Q}=\mathbf{P A R}^{-1}
\end{gathered}
$$

Then, iterate (21)-(22) until $\hat{\mathbf{P}}_{k}$ will not change obviously. Finally, we search the 2-D positions of the spectral peak of $\hat{\mathbf{P}}_{k}$, the coordinates $\boldsymbol{r}$ and $\boldsymbol{\theta}$ of which are the distances and DOA estimates of the targets. $r / c$ are the TOA estimates. The details of SPICE algorithm can be found in [12].

\section{Target localization in underwater environment}

In underwater environment where the seafloor is smooth and the water is stable, the target location can be obtained with TOA and DOA estimates using one sensor array. But there are ocean currents, rocks, and undersea mountains, which may cause multipath and non-line-of- sight (NLOS) propagations and will dramatically degrade the performance of the localization method for LOS conditions. In our previous work [11], a virtual station (VS) method was proposed to solve the NLOS and multipath problems with TOA and DOA measurements. Therefore, it can be directly used in this work when more sensor arrays which can be seen as base stations (BSs) are used. In order to make it simple, the details of the VS method will not be stated in this paper.

To calculate the CRLB of the proposed method, we assume that the underwater environment is simple that the signals undergo no more than two-bound scattering (reflection or diffraction), and multi-bound scattering can be ignored as they suffer from severe fading and may be too weak to be observed.

There are several signal paths between the BS and the target. The first and second detectable paths are utilized in the proposed localization method. We use a new parameter $p_{i}^{k_{i}}$ to represent the probability of that the first or second detectable path is the path $k_{i}\left(k_{i}=1,2, \ldots, K_{i}\right)$ which represents the VS number, where $i=1,2, \ldots, N$ denotes the BS number.

When the underwater signal channel is stable, the first and second detectable paths are stable too. The two path numbers are $q 1$ and $q 2$. We can get

$$
p_{i}^{k_{i}}=\left\{\begin{array}{cc}
1 & k_{i}=q_{1} \text { or } q_{2} \\
0 & k_{i} \neq q_{1} \text { or } q_{2}
\end{array}\right.
$$

When the underwater signal channel is complicated with relatively large flow of fish, the first and second detectable paths may be not the same in every measurement, so $p_{i}^{k_{i}}$ needs to be practically measured.

The probability that the paths $q 1$ and $q 2$ are the first and second detectable paths can be obtained as

$$
P_{i}^{q_{1} q_{2}}=p_{i}^{q_{1}}+p_{i}^{q_{2}}-1
$$

The Fisher information matrix of the proposed method can be given as

$$
\begin{aligned}
\mathbf{J}_{0} & =E\left\{\left[\frac{\partial}{\partial \mathbf{w}} \ln f(\boldsymbol{\Lambda} \mid \mathbf{w})\right]\left[\frac{\partial}{\partial \mathbf{w}} \ln f(\boldsymbol{\Lambda} \mid \mathbf{w})\right]^{T}\right\} \\
& =\sum_{j=1}^{K_{1} ! \times K_{2} ! \times \ldots \times K_{N} !} P_{j}\left[\frac{\partial}{\partial \mathbf{w}} \ln f\left(\boldsymbol{\Lambda}_{j} \mid \mathbf{w}\right)\right]\left[\frac{\partial}{\partial \mathbf{w}} \ln f\left(\boldsymbol{\Lambda}_{j} \mid \mathbf{w}\right)\right]^{T}
\end{aligned}
$$

where

$$
\mathbf{w}=\left[x_{m}, y_{m}\right]^{T}
$$




$$
\begin{aligned}
& P_{j=1}=P_{1}^{1,2} \times P_{2}^{1,2} \times \ldots \times P_{N}^{1,2} \\
& P_{j=2}=p_{1}^{2,1} \times p_{2}^{1,2} \times \ldots \times p_{N}^{1,2} \\
& \quad \ldots \\
& P_{j=K_{1} !=} p_{1}^{K_{1}, K_{1}-1} \times p_{2}^{1,2} \times \ldots \times p_{N}^{1,2} \\
& \quad \ldots \\
& P_{j=K_{1} ! \times K_{2} ! \times \ldots \times K_{N} !}=p_{1}^{K_{1}, K_{1}-1} \times p_{2}^{K_{2}, K_{2}-1} \times \ldots \times p_{N}^{K_{N}, K_{N}-1}
\end{aligned}
$$

$\Lambda$ is the measurement vector with

$$
\begin{aligned}
& \Lambda_{j=1}=\left[\Lambda_{1}^{1,2}, \Lambda_{2}^{1,2}, \ldots, \Lambda_{N}^{1,2}\right] \\
& \Lambda_{j=2}=\left[\Lambda_{1}^{2,1}, \Lambda_{2}^{1,2}, \ldots, \Lambda_{N}^{1,2}\right] \\
& \text {... } \\
& \Lambda_{j=K_{1} !}=\left[\Lambda_{1}^{K_{1}, K_{1}-1} \times \Lambda_{2}^{1,2} \times \ldots \times \Lambda_{N}^{1,2}\right] \\
& \boldsymbol{\Lambda}_{j=K_{1} ! \times K_{2} ! \times \ldots \times K_{N} !}=\left[\Lambda_{1}^{K_{1}, K_{1}-1} \times \Lambda_{2}^{K_{2}, K_{2}-1} \times \ldots \times \Lambda_{N}^{K_{N}, K_{N}-1}\right] \\
& \boldsymbol{\Lambda}=\left[\boldsymbol{\Lambda}_{\mathrm{TOA}}^{T} \mid \boldsymbol{\Lambda}_{\mathrm{DOA}}^{T}\right]^{T} \\
& \Lambda_{\mathrm{TOA} i}=d_{i}+n_{i}=\sqrt{\left(x_{m}-x_{B i}\right)^{2}+\left(y_{m}-y_{B i}\right)^{2}}+n_{i} \\
& \Lambda_{\mathrm{DOA} i}=\theta_{i}+n_{i}=\arctan \frac{y_{m}-y_{B i}}{x_{m}-x_{B i}}+n_{i}
\end{aligned}
$$

The CRLB can be given as

$$
\sigma_{\mathrm{CRLB}}^{2}=\operatorname{tr}\left(\mathbf{J}_{0}^{-1}\right)=\operatorname{tr}\left(\sum_{j=1}^{K_{1} ! \times K_{2} ! \times \ldots \times K_{N} !} P_{j}\left(\mathbf{H}_{j}{ }^{T} \mathbf{Q} \mathbf{H}_{j}\right)^{-1}\right)
$$

where $\mathbf{Q}$ is the covariance matrix and $\mathbf{H}$ is the Jacobian matrix of $\boldsymbol{\mu}(\mathbf{w})$ and can be given as

$$
\mathbf{H}=\frac{\partial \boldsymbol{\mu}(\mathbf{w})}{\partial \mathbf{w}^{T}}
$$

\section{Simulation results}

\subsection{Simulation of TOA and DOA estimation}

We investigate the performance of our algorithm about angular resolution, sidelobe level, and accuracy in the angular spectrum. We also compare it with FrFT-MUSIC algorithm [14] which utilizes MUSIC algorithm combined with FrFT. But in order to apply for passive sonar, the rotation angle $\alpha$ has been changed from $-\operatorname{arccot} \mu$ to $\arctan \mu$.

The chirp signal with a center frequency of $1500 \mathrm{~Hz}$ and a bandwidth of $100 \mathrm{~Hz}$ is used in the simulation. The array contains eight sensors which are uniformly spaced at half wavelength. The number of snapshots is 2 . We consider two targets at $\left(14^{\circ}, 14 \mathrm{~m}\right)$ and $\left(16^{\circ}, 16 \mathrm{~m}\right)$. The noise is assumed to be Gaussian random processes with zero mean and the SNR is $20 \mathrm{~dB}$. The scanning grid for the both algorithms is $1^{\circ}$ and $1 \mathrm{~m}$. The number of iterations is 10 . One hundred independent runs are simulated to obtain the angular spectrum. We can see from Fig. 2a that the FrFT-MUSIC method can approximately detect the targets but it suffers from lower angular resolution than the other two with low snapshots so that it cannot separate the two targets and consider them as one target at $\left(14^{\circ}, 34 \mathrm{~m}\right)$. From Fig. 2b, our FrFT-SPICE algorithm has exactly estimated the TOA and DOA of the two targets and produces the angular spectrum with lower sidelobe levels, higher angular resolution, and higher accuracy in low snapshots conditions.

\subsection{Simulation of the VS method}

The simulations in our previous work [11] have already demonstrated the effectiveness of the proposed VS

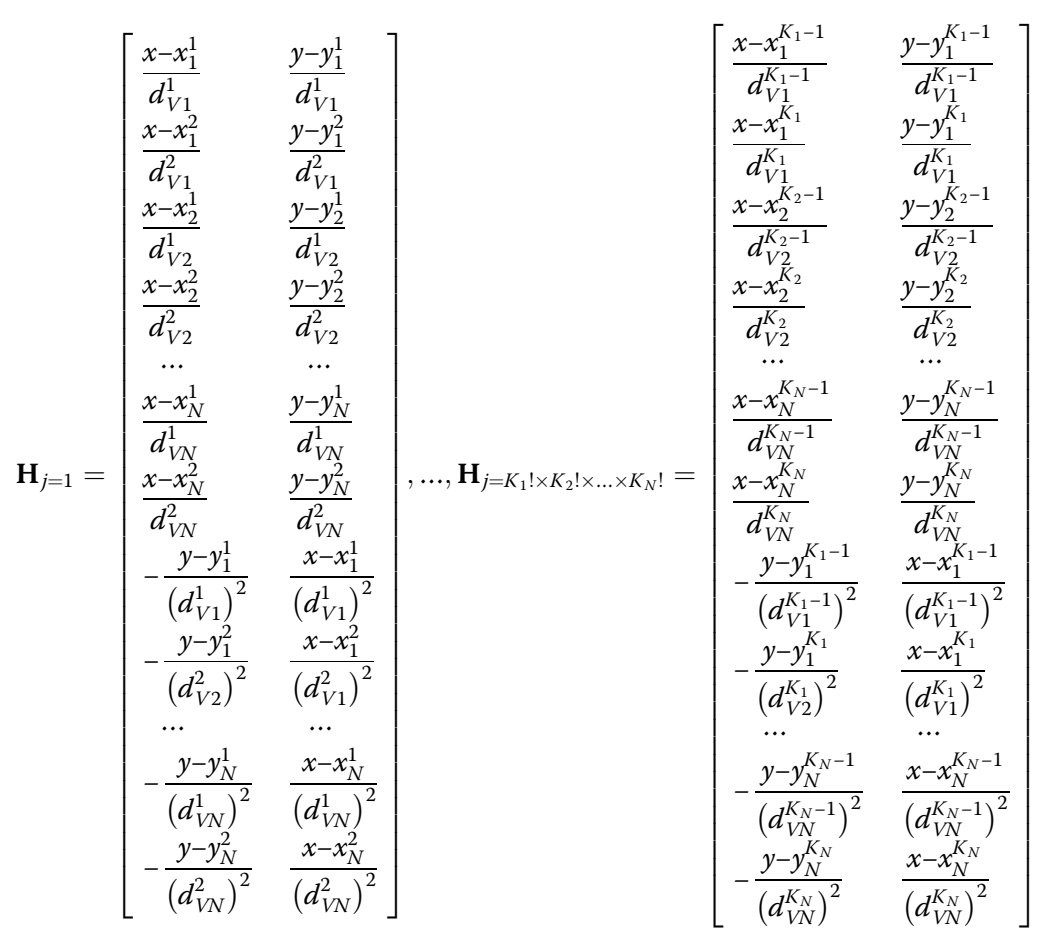




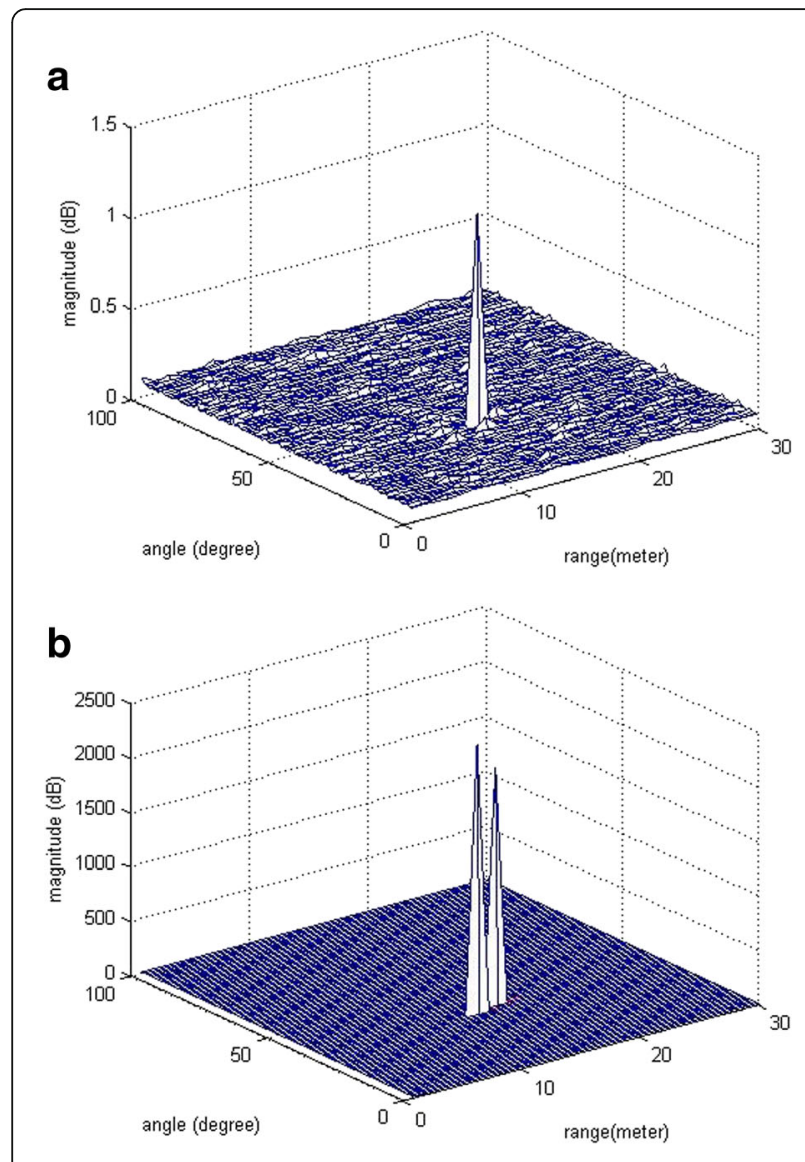

Fig. 2 Comparison of the angular spectrum. a FrFT-MUSIC. b FrFT-SPICE

method for the NLOS and multipath underwater environments, so there is no need to simulate it again in this paper. The CRLB of the proposed VS method which has not been analyzed in previous works will be simulated in this section.

The CRLB in Fig. 3 is obtained in the same situations as in [11]. The target is located at three different places at $\mathrm{A}, \mathrm{B}$, and $\mathrm{C}$ where the multipath problem becomes more and more complicated. Most of the paths are direct paths when the target is located at point A. At point $\mathrm{B}$, all paths are one-bound scattering paths. At point $\mathrm{C}$, almost all paths are multiple-bound scattering paths. We assume that $p_{i}^{1}=p_{i}^{2}=\ldots=p_{i}^{K_{i}}$. It can be seen that our VS method is close to the CRLB, but the distance between the RMSE and the CRLB has a slow increase as the multipath conditions become more serious.

\section{Conclusions}

This paper has presented a nearfield target localization method for sonar system which uses wideband chirp signal. In underwater conditions, the signal channel is not stable and there exists multipath problem which can cause large localization errors when the traditional
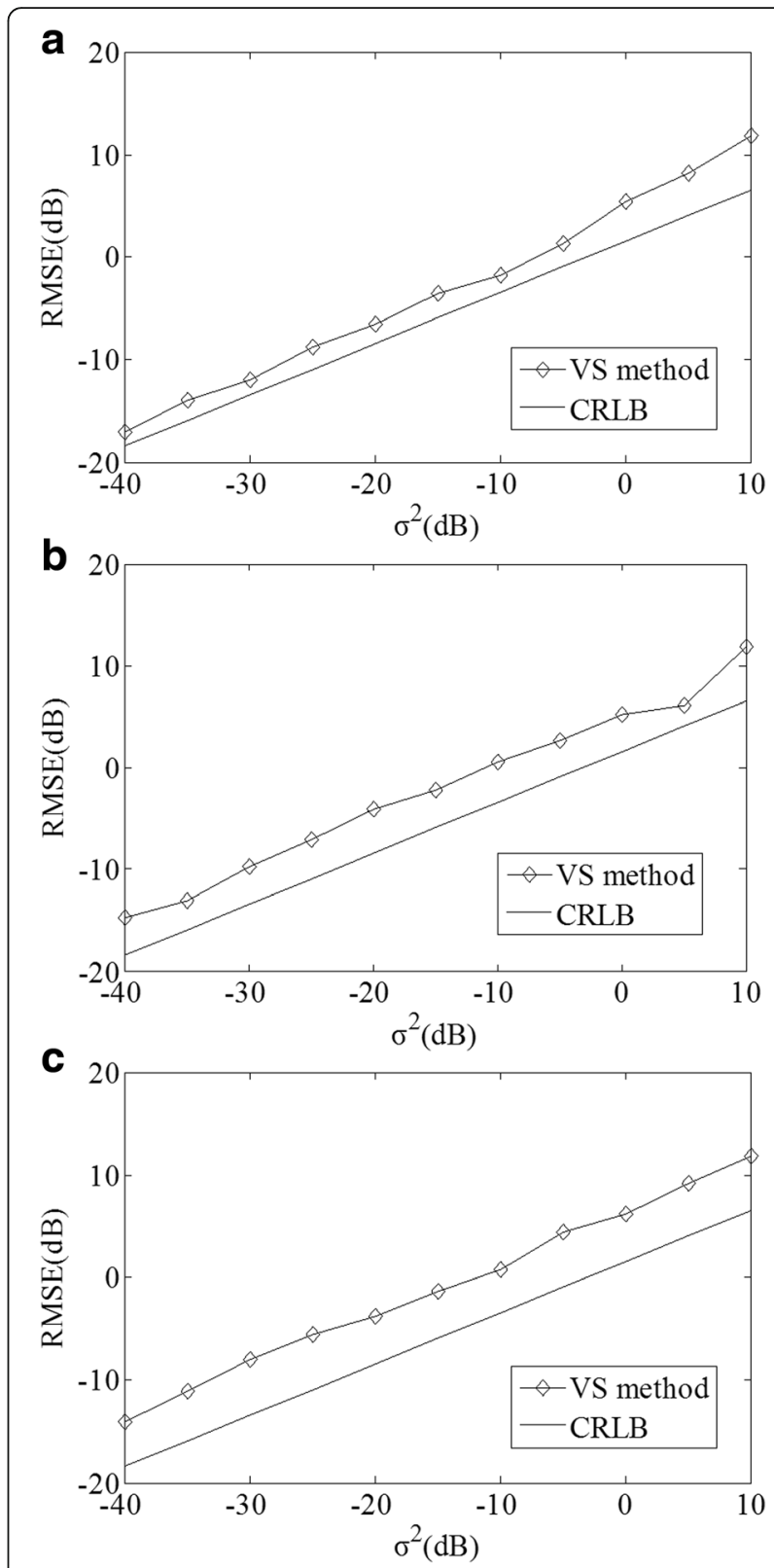

Fig. 3 CRLB of the VS method. a Point A. b Point B. c Point C

localization methods are used. We extend the SPICE algorithm in the FrFT domain so that the TOA and DOA of wideband chirp signals can be estimated with a few snapshots. The proposed method has high angular resolution and low sidelobe levels. We also utilize the proposed VS method to solve the multipath problem. The simulation results have demonstrated the effectiveness of the proposed method.

\section{Abbreviations}

BS: Base station; CRLB: Cramer-Rao lower bound; DOA: Direction of arrival; FRFT: Fractional Fourier transform; LOS: Line-of-sight; NLOS: Non-line-of-sight; TOA: Time of arrival; VS: Virtual station 


\section{Funding}

The experiments of this research are funded by the National Natural Science Foundation of China under Grant numbers 61601494 and 61602346.

\section{Authors' contributions}

All authors contributed to the work. DL conceived and designed the study. ZL carried out most of the analyses. DL drafted the manuscript. XG and PH performed the experiments. All authors read and approved the final manuscript.

\section{Competing interests}

The authors declare that they have no competing interests.

\section{Publisher's Note}

Springer Nature remains neutral with regard to jurisdictional claims in published maps and institutional affiliations.

\section{Author details}

'Tianjin Key Laboratory of Wireless Mobile Communications and Power Transmission, Tianjin Normal University, Tianjin 300387, China. ${ }^{2}$ College of Electronic and Communication Engineering, Tianjin Normal University, Tianjin 300387, China. ${ }^{3}$ Army Engineering University, Shijiazhuang 050003, China.

Received: 5 November 2017 Accepted: 11 April 2018

Published online: 27 April 2018

\section{References}

1. Z Li, K Liu, Y Zhao, et al., MaPIT: an enhanced pending interest table for NDN with mapping bloom filter. IEEE Commun. Lett. 18(11), 1915-1918 (2011).

2. Z Li, Y Chen, H Shi, et al., NDN-GSM-R: a novel high-speed railway communication system via named data networking. EURASIP J. Wirel. Commun. Netw. 48, 1-5 (2016).

3. X Liu, Z Li, P Yang, et al., Information-centric mobile ad hoc networks and content routing: a survey. Ad Hoc Netw. (2016) https://doi.org/10.1016/j. adhoc.2016.04.005.

4. Z Z Li, L Song, H Shi, et al., Approaching the capacity of k-user MIMO interference channel with interference counteraction scheme. Ad Hoc Netw. (2016) https:/ doi.org/10.1016/j.adhoc.2016.02.009.

5. Z Li, Y Chen, K Liu, Hybrid wireless networks with FIB-based named data networking. EURASIP J. Wirel. Commun. Netw. 54, 1-7 (2017).

6. Z Li, Y Xu, K Liu, Performance analysis of a novel $5 \mathrm{G}$ architecture via contentcentric networking. Phys. Commun. (2017) https://doi.org/10.1016/.jphycom. 2017.04.002

7. CK Seow, SY Tan, Non-line-of-sight localization in multipath environments. IEEE Trans. Mob. Comput. 7(5), 647-660 (2008).

8. YT Ma, LJ Zhou, KH Liu, Iterative phase reconstruction and weighted localization algorithm for indoor RFID-based localization in NLOS environment. IEEE Sensors J. 14(2), 597-611 (2014).

9. CS Tai, SY Tan, CK Seow, Robust non-line-of-sight localization system in indoor environment. Electron. Lett. 46(8), 593-595 (2010).

10. CK Seow, SY Tan, Non-line-of-sight unidirectional mobile localization in multipath environment. Electron. Lett. 44(2), 141-142 (2008).

11. D Liu, K Liu, Y Ma, J Yu, Joint TOA and DOA localization in indoor environment using virtual stations. IEEE Commun. Lett. 18(8), 1423-1426 (2014).

12. P Stoica, P Babu, J Li, New method of sparse parameter estimation in separable models and its use for spectral analysis of irregularly sampled data. IEEE Trans. Signal Process. 59(1), 35-46 (2011).

13. Z Chen, J Li, P Stoica, KW Lo, in Proc. IEEE OCEANS. Iterative adaptive approach for wide-band active sonar array processing (Sydney, IEEE, 2010), pp. 1-10.

14. P Luo, K Liu, W Shi, G Yan, in Proc 10th ICSP. 2-D DOA estimation of wideband LFM signals for arbitrary planar array (2010), pp. 307-310.

15. HM Ozaktas, O Arikan, MA Kutay, Digital computation of the fractional Fourier transform. IEEE Trans. Signal Process. 44(9), 2141-2150 (1996).

16. JD Scargle, Studies in astronomical time series analysis. II. Statistical aspects of spectral analysis of unevenly spaced data. Astrophys. J. 263, 835-853 (1982).

\section{Submit your manuscript to a SpringerOpen ${ }^{\circ}$ journal and benefit from:}

- Convenient online submission

Rigorous peer review

- Open access: articles freely available online

- High visibility within the field

- Retaining the copyright to your article

Submit your next manuscript at $\boldsymbol{\nabla}$ springeropen.com 